\title{
A Framework for Facial Surgery Simulation
}

\author{
R. M. Koch, S. H. M. Roth, M. H. Gross, ${ }^{*}$ A. P. Zimmermann, H. F. Sailer ${ }^{\dagger}$ \\ * Computer Science Department, ETH Zurich, Switzerland \\ ${ }^{\dagger}$ Sailer Clinic, Zürich, \\ e-mail: $\{$ koch, roth, grossm $\} @$ inf.ethz.ch
}

\begin{abstract}
The accurate prediction of the post-surgical facial shape is of paramount importance for surgical planning in facial surgery. In this paper we present a framework for facial surgery simulation which is based on volumetric finite element modeling. We contrast conventional procedures for surgical planning against our system by accompanying a patient during the entire process of planning, medical treatment and simulation. In various preprocessing steps a 3D physically based facial model is reconstructed from CT and laser range scans. All geometric and topological changes are modeled interactively using Alias. ${ }^{\mathrm{TM}}$ Applying fully $3 \mathrm{D}$ volumetric elasticity allows us to represent important volumetric effects such as incompressibility in a natural and physically accurate way. For computational efficiency, we devised a novel set of prismatic shape functions featuring a globally $C^{1}$-continuous surface in combination with a $C^{0}$ interior. Not only is it numerically accurate, but this construction enables us to compute smooth and visually appealing facial shapes.
\end{abstract}

Keywords: Finite Element Method, Facial Surgery Simulation, Facial Modeling, Data Reconstruction.

\section{INTRODUCTION}

\subsection{Background}

There is a wide spectrum of facial malformations and diseases which maxillofacial and craniofacial surgeons have to take care of. This includes but is not restricted to diseases like injuries and tumors as well as deformities due to inherited syndromes (e.g. Crouzon-Syndrome, Apert-Syndrome) or developmental disorders (e.g. disturbed growth of the jaw). For patients seeking for surgical treatment in order to correct such malformations it would be of high benefit to have a means to predict the post-surgical appearance of their face in a reliable way. Further, facial surgery has to strive for the reconstruction of a balanced face as even very fine variations of facial proportions can affect the appearance of a face strongly and thus distort its harmony [10].

The prediction and planning of surgical procedures to correct such aberrant skeletal anatomy can to date only be performed in a two-dimensional way from one single perspective, most often the profile view of the patient, such as illustrated in the top row of figure 2. All other views only can be estimated roughly.

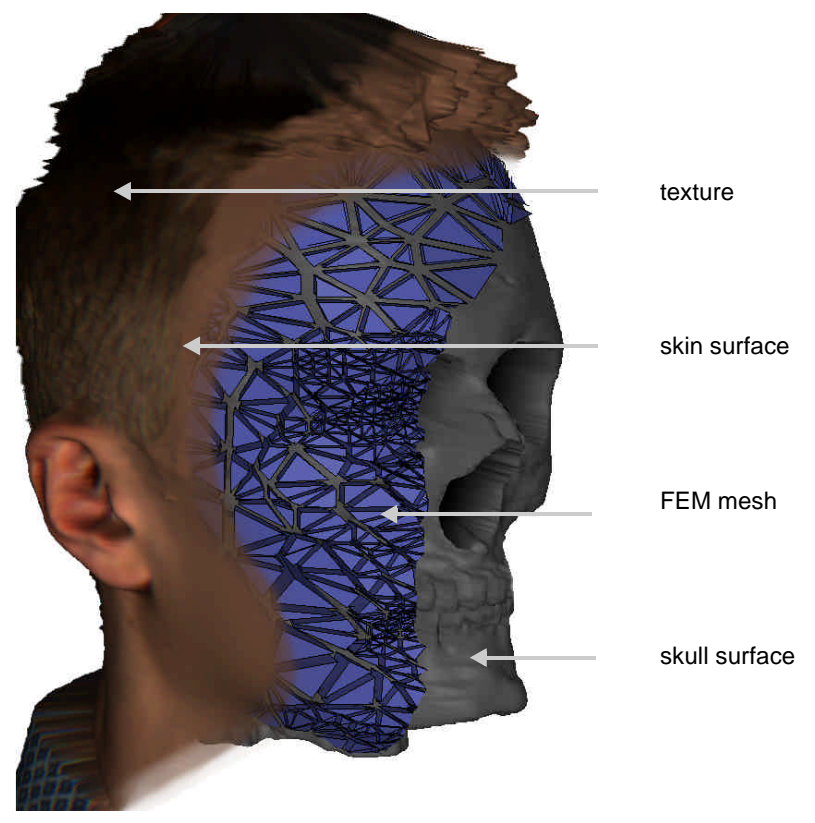

Figure 1: Illustration of the physically based model

Hence, the fullness of the lips, the width of the nose, the width and projection of the cheekbones and the influence of the surgery on the appearance of the eyes cannot be predicted. For the time being, only the surgeon's experience gives information on the overall outcome of the patients facial appearance (see e.g. [7, 3]).

Therefore, both surgeon and patient have a strong need for a method which enables them to compute highly realistic pictures of the expected post-surgical shape during the planning of a surgical procedure.

\subsection{Previous Work}

The field of facial modeling has been an area of growing research efforts for more than a decade. First approaches such as [15] were based on geometric deformations using parametric surfaces and aimed primarily at facial animation. Later, physically based simulation paradigms were adopted in order to model more accurately the physical properties of elastic materials (see e.g. [21]). For a survey of facial animation see e.g. [15].

Back in 1986, Larrabee [11] stated a finite element model of skin deformation. This work was followed by Deng's Ph.D thesis [5], where she presented an analysis of plastic surgery by means of the finite element method. In 1991, Pieper [17] summed up his efforts to provide a system for computer-aided plastic surgery in his Ph.D thesis. To our knowledge, this is the first time that facial simulation in combination with finite element modeling was employed as a means of planning surgical procedures. He focussed on plastic surgery and therefore concentrated on cutting and stretching of skin and epidermis rather than on repositioning bones. Further, his model lacked the resolution required for a reliable simulation of very subtle changes in the appearance of a face and did not provide a $C^{1}$-continuous surface.

Lee et al. [12] presented a promising approach to facial animation where they introduced a layered tissue model based on masses and springs connected to form prism-shaped elements. The facial model is adapted from a template face, takes into account various anatomical aspects and aims at facial animation. Koch et al. [10] proposed a method which provides a $C^{1}$-continuous finite element surface connected to the skull by springs. This model is generated directly from individual facial data sets and has successfully been tested for surgery simulation and emotion editing [10] on the Visible Human Data Set. Although providing very promising results the model lacks true volumetric physics. 
Therefore, in the field of surgery simulation, attention focussed on the development of volume-based models in combination with more and more sophisticated finite element solution schemes. Keeve et al. [9] presented a system for facial surgery simulation combining Lee's layered tissue model with a finite element approach in order to solve the inherent partial differential equations. Another interesting approach aiming at real-time applications was proposed by Bro-Nielsen et al. [2]. Both methods only made use of linear interpolation within the elements and therefore suffer from $C^{0}$ artifacts both on the surface and within the volume.

Recently, Roth [19] presented a versatile framework for the finite element simulation of soft tissue using tetrahedral Bernstein-Bézier elements. They incorporated higher order interpolation as well as incompressible and nonlinear material behavior, but again restricted themselves to $C^{0}$-continuous interpolation across element boundaries.

Apart from the limitations mentioned above, all previous approaches lack an elaborate validation and error analysis with respect to craniofacial surgery.

\subsection{Our Approach}

To optimize both accuracy and rendering quality our goal was to combine the physical correctness of volumetric finite element simulation with the superior quality of the $C^{1}$-continuous surface of $[4,10]$. Furthermore, a validation of the proposed model will investigate its applicability to facial surgery simulation.

As a first major contribution we therefore extended the surface-based approach of [10] to volumetric physics which involved both the reformulation of the mathematical and physical foundations and the redesign of the special purpose triangular finite element model of the human face. As a result we devised prism shaped volumetric elements an illustration of which is given in figure $7 \mathrm{a}$. While still providing a $C^{1}$-continuous surface using a reduced number of degrees of freedom this volumetric model features a more accurate simulation of tissue behavior including volume preservation and pressure calculations.

The second contribution is an evaluation of our approach with a group of test patients, two of them are discussed in the result section. To achieve this, a prototype application was built which is based on data available from individual patients in a clinical environment. This includes CT scans of the pre- and post-surgical situation in combination with high resolution laser range surface scans. Both individual parameters, such as different stiffness and elasticity of tissue, and the exact bone movements introduced by the surgeon are taken into account in order to simulate as accurately as possible the outcome of real surgery. With validation in mind we re-simulate the surgical procedures carried out on the test group of patients with cranio-maxillofacial abnormities. The simulation of bone movements is accomplished by means of the commercially available modeling system Alias. ${ }^{\mathrm{TM}}$

The outline of the paper is as follows: throughout the report we will accompany a patient who underwent both the conventional and the envisioned treatment before and after the surgical procedure. Section Section 1.4 describes the process of medical treatment beginning with the first consultative meeting of patient and surgeon. We then compare the conventional and new planning sequence of the surgical procedure. After surgery, the treatment ends with several follow-up checks. An overview of the model build-up and simulation is given in section Section 2 . Section Section 3 discusses the finite element approach including a brief review of the physical foundations and the design of the shape functions. A quantitative evaluation of our method is given in section Section 4.

\subsection{Accompanying a Patient}

The patient accompanied through the following sections is suffering from a so-called short face with a deep bite caused by the retropositioned mandible, i.e. the lower jaw, as well a reduced vertical facial height due to a maxilla positioned to high. Figure 1 illustrates the corresponding physically based model the construction of which will be described in section Section 2 .

We will compare the different steps in data acquisition as well as differences in the planning set-up and post-surgical treatment. A general view of the major phases of treatment including timing information can be found in figure 2. The top row contrasts the conventional to the envisioned setting presented in the bottom row.

In both cases the treatment begins with a consulting phase during which patient and surgeon discuss the details of the treatment. The decision, if orthodontic correction of the teeth is nec-

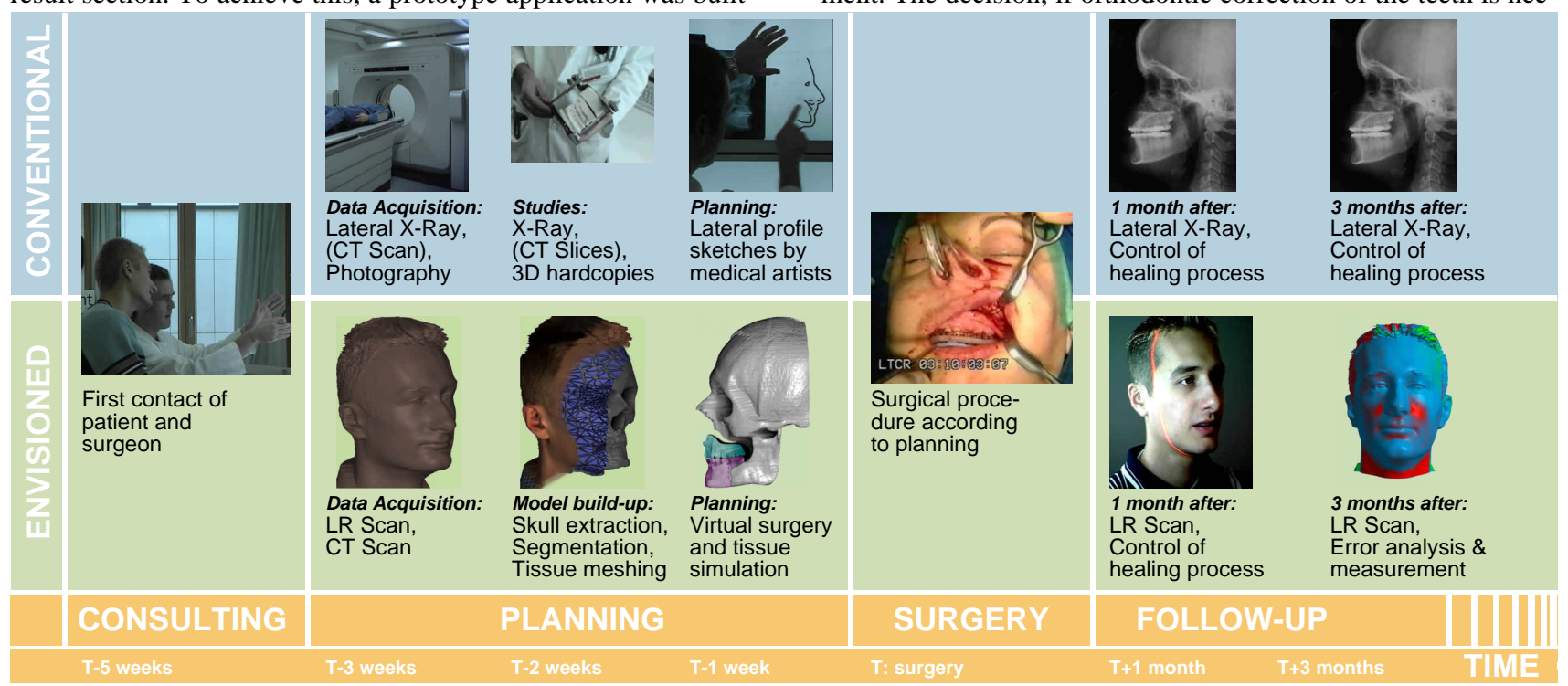

Figure 2: Time-line of a patient from first consultations to recovery. The top row represents the conventional sequence and is contrasted against the envisioned virtual surgery planning framework shown in the bottom row. 
essary has to be made during this phase. In case of a surgical procedure, considerations about the required bone movements and expected convalescence time follow. Afterwards, as a first step of the planning and preparation of surgery, the data acquisition follows. In both cases this comprises lateral and frontal photographs of the pre-surgical situation and the acquisition of a CT scan, if needed. The volume data set can be used to build 3D hardcopies of bone structures on the one hand, and serves as an integral component of the facial model used to simulate the surgery on the other hand (see section Section 2). In addition to the data mentioned above the envisioned planning approach requires highly accurate surface laser range scans which are also incorporated into the model underlying the finite element simulation outlined in section Section 3.

In the conventional setting, the planning mainly consists of structured analyzing lateral X-Ray images upon which studies of the actual and envisioned profile can be sketched. These sketches in combination with the surgeon's experience allow the estimation of bone movements and cuts necessary to correct the disfigurement. Corresponding linear displacements will now be transferred to plaster-cast models of the maxillomandibular complex (lower and upper jaw) for surgery simulation.

In the envisioned setting, the data acquisition phase is followed by the model build-up which consists of skull and surface extraction, tissue segmentation, and mesh generation. This process and the modeling of the surgical procedure on this model will be described in detail in section Section 2.

A convalescence and recovery of four to six weeks and a follow-up time of up to six or even nine months follows the operative treatment. In addition to the medical controlling of the healing process in several follow-up checks, laser range scans of the post-surgical facial surface enable to compare the correspondence of simulation and the real outcome of surgery. Furthermore, the process of detumescence (diminution of swelling) can be documented to a new level of accuracy.

\section{MODELING OF SURGERY}

In this section, both the construction of the facial model and the simulation of the surgical procedure are outlined. We focus on the data and algorithms used to build up the model as well as on the definition of bone movements which correspond to the actual surgery.

\subsection{Model build-up}

The model underlying to the finite element approach must be built upon data available from individual patients including CT and laser range scans (LR). After the acquisition of data, the following steps must be performed in order to arrive at a facial model capable of capturing the basic properties of tissue and offering the instrumentation for surgical simulation at sufficient accuracy.

\section{Registration of Volume and Surface Data}

In a first step, volume (CT) and surface (LR) data must be registered in a common coordinate frame. To achieve this we extract the facial surface in the CT scan using the marching cubes algorithm [14]. The problem now reduces to finding a transformation which maps the LR surface onto the surface originating form the isosurface extraction. Due to the fact that the scaling of each surface is determined by the scanning method, we are left with finding only a suitable translation and rotation in order to register the geometries. To this aim, we start by manually setting landmark pairs on both surfaces. These landmarks are selected to represent characteristic and easy to locate facial features, as e.g. the corners of the eyes or the mouth and the tip of the nose.

Starting from manually prematched geometries to avoid local minima, the registration process can be regarded as the minimization of a scalar error function $E$ defined by the square distances of $n$ corresponding landmark points $\boldsymbol{u}_{i}$ and $\boldsymbol{u}_{i}^{\prime}$. E depends on the rotation $\boldsymbol{r}$ around the three axes as well as on the translation vector $\boldsymbol{t}$.

$$
\begin{aligned}
& E(\boldsymbol{r}, \boldsymbol{t})=\sum_{i=1}^{n}\left(\mathbf{M} \boldsymbol{u}_{i}-\boldsymbol{u}_{i}^{\prime}\right)\left(\mathbf{M} \boldsymbol{u}_{i}-\boldsymbol{u}_{i}^{\prime}\right)^{T}, \\
& \mathbf{M}=\left[\begin{array}{cc}
\mathbf{M}_{\mathrm{rot}}(\boldsymbol{r}) & \boldsymbol{t} \\
\mathbf{0} & 1
\end{array}\right],\left(\mathbf{M}_{\mathrm{rot}}(\boldsymbol{r}) \text { : Rotation corresponding to } \boldsymbol{r} \text {. }\right)
\end{aligned}
$$

For minimizing $E(\boldsymbol{r}, \boldsymbol{t})$ we employ a method of conjugate directions. It is a slightly modified version of Powell's algorithm which is presented in [18].

\section{Mesh Generation}

The next step consists of the mesh generation for the finite element engine. For this purpose we decimate the LR surface mesh using the approach of Schroeder et al. [20] in combination with local Delaunay re-triangulations.

After transforming the reduced mesh according to the matrix computed in the registration step, the facial tissue is tiled with prism shaped elements. We follow the approach of Waters [22] which in essence performs a cylindrical projection of every vertex of the decimated mesh onto the skull inside the CT data set.

\section{Assignment of Material Parameters}

After the mesh generation we have to assign material parameters in accordance with the CT data to the elements. This is accomplished by a segmentation of CT data into four distinct regions: skin, fat, muscle, and bones. In order to achieve this segmentation we isolate four training regions which are known to belong to one of the above categories. The averages of their CT values provide centroids for further segmentation. In addition, each tissue type is assigned both a Young's modulus E, defining its elasticity and a Poisson's ratio $v 0 \leq v \leq 0.5$ which describes its compressibility. The assignment of such values is done according to [11]. Linear interpolation between the centroids provides us with intermediate values both for $E$ and $v$ for all voxels of the $\mathrm{CT}$ data set. Figure 3 shows a schematic overview of the procedure.

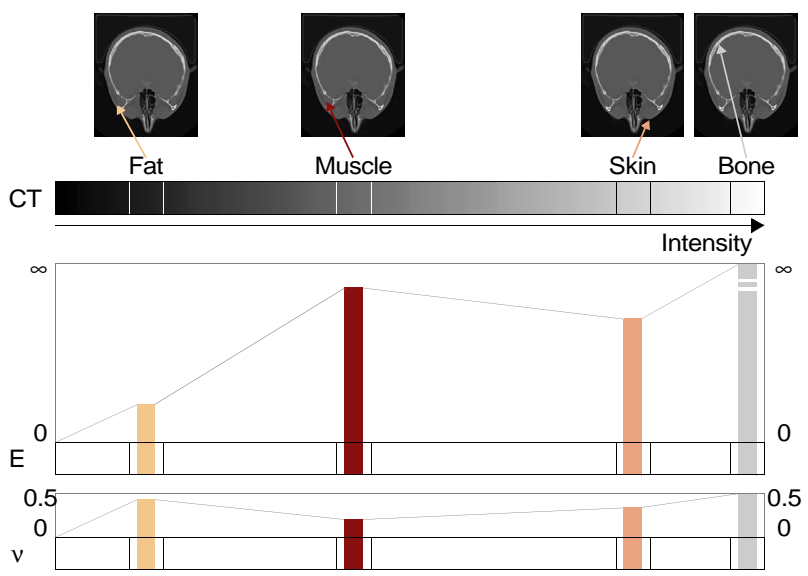

Figure 3: Schematic view of the process of CT segmentation and corresponding parameter assignment 
It has to be stated, that $E$ and $v$ highly depend on a patient's gender, age and other parameters. Consequently, the problem of measuring and assignment of accurate soft tissue material parameters is still subject to current research in the field of biomedicine. However, the prescribed displacement approach (see sections Section 2.2 and Section 3) we employ for the FEM computations makes the system robust against variations of $E$ and $v$.

After having determined $E$ and $v$ for each CT voxel we still have to assign them to the prism elements resulting from the mesh generation step. This is accomplished by averaging $E$ and $v$ over all the voxels interior to the corresponding prism using a 3D scan conversion algorithm.

\subsection{Modeling the Surgical Procedure}

In order to simulate a surgical procedure we model the bone cuts (osteotomies) and bone movements (e.g. advancement of the jaw bone) with the help of a craniofacial surgeon using the Alias $^{\mathrm{TM}}$ modeling system. Figure 4 depicts the advancements performed on upper and lower jaw bone structures of our example patient.

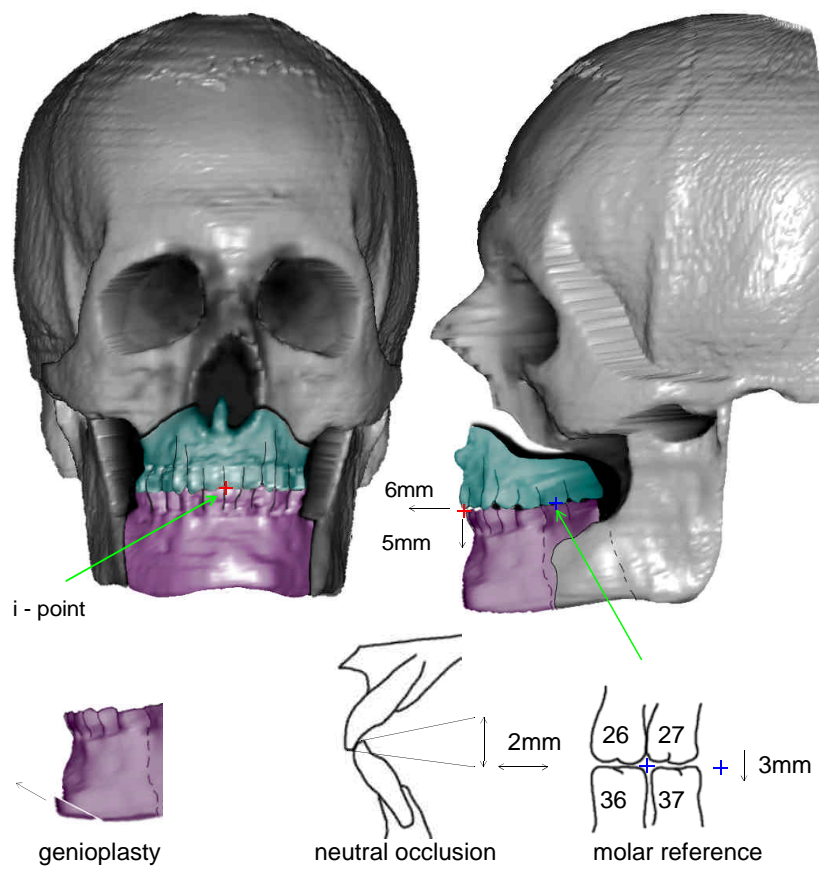

Figure 4: Reference points used to model craniofacial surgery with the Alias ${ }^{\mathrm{TM}}$ modeling system

In order to reposition the upper jaw three reference points are used: the $i$-point, situated between the tips of the upper incisors, and either the tips of the upper canines or the molars 17 and 27 on the right and left side of the upper jaw respectively. The tooth numbering in figure 4 refers to the standard of the World Dental Federation (FDI). The lower jaw is repositioned with regard to the upper jaw in order to reach a neutral occlusion of two millimeters. In some cases an additional genioplasty is indicated where a part of the lower jaw is cut and moved according to figure 4 .

Knowing corresponding vertices of pre- and post-surgical skull surface makes it possible to calculate the displacement field which will be input to the finite element engine as prescribed displacement boundary conditions (see section Section $3)$.
In a second step, we determine regions which will not be changed during the surgical procedure and therefore can serve as additional zero displacement boundary conditions. This is accomplished by using the 3D paint program StudioPaint ${ }^{\mathrm{TM}}$ which allows one to draw directly onto a three-dimensional surface. Figure 5 depicts an example setting of the boundary conditions both on the skull and on the facial surface.
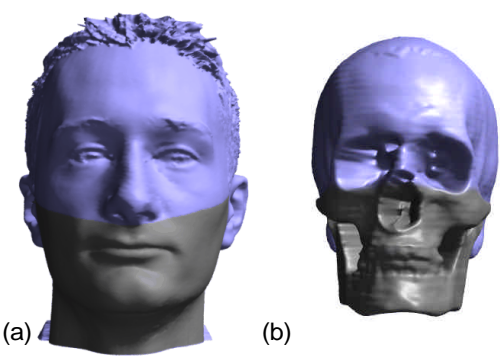

(b)

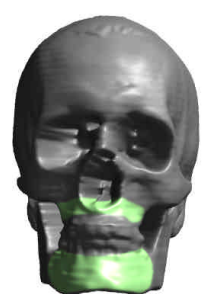

Figure 5: Boundary conditions:

(a) zero displacement boundary conditions on the facial surface

(b) zero displacement boundary conditions on the skull

(c) prescribed displacement boundary conditions on the skull

\section{FEM MODEL}

This section introduces the finite element system employed for surgery simulation. For reasons of readability we briefly review the basic notions of static elastomechanics which are fundamental for the subsequent discussion of the volumetric FEM approach. Then we elaborate the construction scheme for the hybrid $C^{0} / C^{1}$-continuous volume interpolation functions designed for our framework. A section on matrix formulation give a recipe for the FEM implementation. All mathematical formulations in this section closely follow the notation of [1].

\subsection{Static Elastomechanics}

The soft tissue model we use for facial surgery simulation requires the following idealizations:

- Rather than by explicit application of external body $\left(\mathbf{f}^{B}\right)$ or surface $\left(\mathbf{f}_{f}^{\boldsymbol{S}_{f}}\right)$ loading forces soft tissue deformations are invoked by so-called prescribed skull displacements obtained from the surgical procedure (see figure $5 \mathrm{c}$ ). Using this approach reduces so-called locking effects [1].

- We restrict our model to the laws of linear elasticity, since the displacements and deformations in most craniofacial operations are small in an FEM sense. In addition, we assume the elasticity as being constant, i.e. independent of the stress.

- Tissue parameters like elasticity and incompressibility do not vary throughout an element.

We think of an elastic body $\mathbf{B}$ - the pre-surgical face - in a cartesian $X, Y, Z$ coordinate system as depicted in figure 6 . The

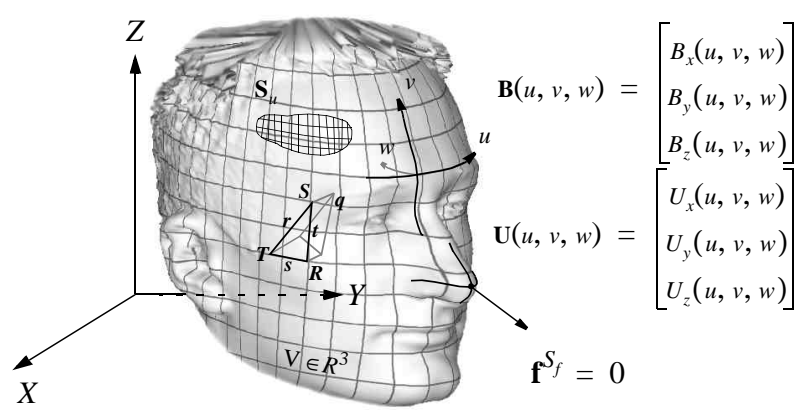

Figure 6: Three-dimensional face with one example 6-node prism element

volume is parametrized in $u$ and $v$ over the surface and in $w$ 
towards the skull. The computational problem consists of finding the displacement function $\mathbf{U}$ which describes the displacement resulting from facial surgery at each point of the volume $\mathbf{B}$. In our model this function is a priori known at rigid parts of the face and skull (zero displacement boundary conditions $\mathbf{S}_{u}$ ) as well as on the parts of the skull that were moved during the surgical procedure (prescribed displacement boundary conditions).

We consider the strain $\varepsilon$

$$
\varepsilon=\left[\varepsilon_{X X} \varepsilon_{Y Y} \varepsilon_{Z Z} \gamma_{X Y} \gamma_{Y Z} \gamma_{Z X}\right]^{T}
$$

which is defined by first order derivatives of the displacement field $\mathbf{U}$. The first three components are known as the volumetric strains

$$
\varepsilon_{X X}=\frac{\partial U_{x}}{\partial X}, \quad \varepsilon_{Y Y}=\frac{\partial U_{y}}{\partial Y}, \quad \varepsilon_{Z Z}=\frac{\partial U_{z}}{\partial Z}
$$

whereas the second three components are denoted as the deviatoric strains

$$
\gamma_{X Y}=\frac{\partial U_{x}}{\partial Y}+\frac{\partial U_{y}}{\partial X}, \quad \gamma_{Y Z}=\frac{\partial U_{y}}{\partial Z}+\frac{\partial U_{z}}{\partial Y}, \quad \gamma_{Z X}=\frac{\partial U_{z}}{\partial X}+\frac{\partial U_{x}}{\partial Z}
$$

The strain caused by the displacement results in a corresponding stress $\tau$

$$
\tau=\left[\tau_{X X} \tau_{Y Y} \tau_{Z Z} \tau_{X Y} \tau_{Y Z} \tau_{Z X}\right]^{T} .
$$

For small strains the fundamental relationship between $\tau$ and $\varepsilon$ is established by the constitutive relation

$$
\tau=\mathbf{C} \varepsilon .
$$

C depends on the elasticity $E$ of the material and on its incompressibility v, denoted by Young's modulus and Poisson ratio, respectively. The exact definition can be found in [1].

The Poisson ratio varies between zero for fully compressible and 0.5 for fully incompressible materials. Note that the coefficients of $\mathbf{C}$ increase with $v \rightarrow 0.5$ and, consequently, $\mathbf{C}$ is not defined for $v=0.5$. For the simulation of incompressibility used to model volume preservation we therefore have to use a different formulation which will be described in the next section.

The solution $\mathbf{U}$ of the problem is the configuration with minimal potential energy which corresponds to the equilibrium of internal elastic energy and the work done by external forces. The absence of such forces allows one to establish the equilibrium condition as a zero virtual work which can be formulated as

$$
\int_{V} \bar{\varepsilon}^{T} \tau d V=0 \Leftrightarrow \int_{V} \bar{\varepsilon}^{T} \mathbf{C} \varepsilon d V=0
$$

The overbar denotes virtual strains caused by virtual displacements. Further mathematical elaborations on the notion of virtual work are omitted for brevity. A detailed discussion can be found in [1].

\subsection{Mixed Formulation}

In order to deal with incompressible materials the strain has to be separated into its volumetric and deviatoric components and in addition to the displacement function $\mathbf{U}$ the pressure $p$ needs to be introduced as an additional variable. This approach is termed the finite element mixed formulation [1] and will briefly be reviewed in the following.
The constitutive equation (6) can be reformulated using indicial notation by separating volumetric and deviatoric components

$$
\begin{aligned}
& \text { volumetric part } \\
& \tau_{i j}=\overbrace{\kappa \varepsilon_{V} \delta_{i j}}+\underbrace{2 G \varepsilon_{i j}^{\prime}} \quad, i, j \in\{X, Y, Z\}
\end{aligned}
$$

deviatoric part

with the new tissue parameters bulk modulus $\kappa$ and shear modulus $G$ which follow from $E$ and $v$ to

$$
\kappa=\frac{E}{3(1-2 v)} \text { and } G=\frac{E}{2(1+v)} .
$$

Further in (8), $\delta_{i j}$ is the Kronecker delta, while $\varepsilon^{\prime}$ and $\varepsilon_{V}$ denote the deviatoric and volumetric strain respectively:

$$
\begin{gathered}
\varepsilon_{i j}^{\prime}=\varepsilon_{i j}-\frac{\varepsilon_{X X}+\varepsilon_{Y Y}+\varepsilon_{Z Z}}{3} \delta_{i j}, \\
\varepsilon_{V}=\varepsilon_{X X}+\varepsilon_{Y Y}+\varepsilon_{Z Z} \approx \frac{\Delta V}{V} .
\end{gathered}
$$

The volumetric strain approximates the proportional change of the volume of the body denoted by $\frac{\Delta V}{V}$. Relating a change in volume to a change in pressure we find

$$
p=-\kappa \varepsilon_{V},
$$

which can be used to reformulate (8) as

$$
\tau_{i j}=-p \delta_{i j}+2 G \varepsilon_{i j}^{\prime}
$$

Note that for fully incompressible materials $\kappa$ in (12) is infinite but the pressure $p$ is still defined.

Hence, the virtual work of (7) converts to

$$
\int_{V} \bar{\varepsilon}^{T} 2 G \varepsilon^{\prime} d V-\int_{V} \bar{\varepsilon}_{V} p d V=0
$$

In order to establish a relationship between the independent variables pressure and displacement in (14) we introduce (12) written in integral form as a second equation:

$$
\int_{V}\left(\frac{p}{\kappa}+\varepsilon_{V}\right) \bar{p} \mathrm{dV}=0
$$

We can think of $\bar{p}$ in (15) as a Lagrange multiplier enforcing the constraint (12) between pressure and displacement.

If both equation (14) and (15) are fulfilled the displacement field $\mathbf{U}$ provides a solution to the problem and the resulting postsurgical face is given by $\mathbf{B}^{\prime}=\mathbf{B}+\mathbf{U}$.

In the following section we derive a set of interpolation functions which will be used to expand the solution $\mathbf{U}$ within a finite element in a Galerkin type approach to discretize (14) and (15).

\subsection{Interpolation Functions}

The design of the volumetric interpolation functions used in our approach was motivated both by the demand to conform to the underlying physics and by the need for visually appealing facial surfaces. In order to satisfy both aims we come up with a set of prismatic interpolation functions featuring $C^{1}$-continuity at the facial surface. Both the interior and the boundary triangle at the bottom - representing a patch of the skull surface - are left to be $C^{0}$-continuous in order to reduce the overall number of degrees 
of freedom (DOF) of the system. The prismatic topology of the elements specifically simplifies the FEM mesh generation. The element and all DOFs are depicted in figure 7.

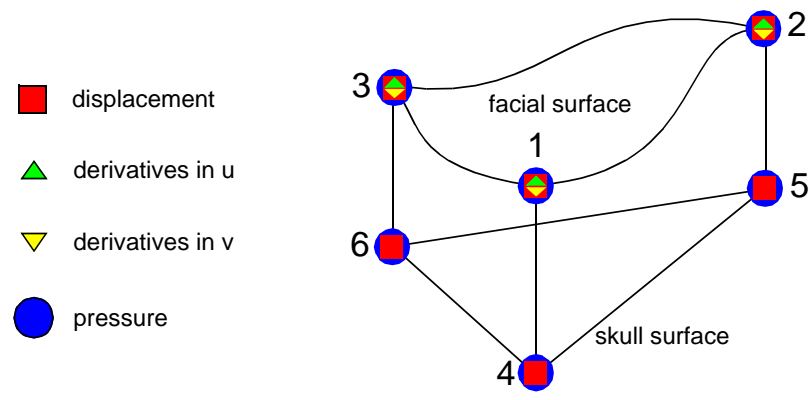

Figure 7: Proposed prism element

In the following sections we step by step derive a set of twelve functions that feature the required $C^{1}$-continuity at the prism surface. The major advantage of these functions compared to a straightforward tensor product extension of the triangle surface shape functions presented in [4] and [10] is that they achieve the desired smoothness at the surface with only nine DOFs instead of twelve.

\section{Trivariate $C^{0}$ Shape Functions}

Let $R, S$, and $T$ define a barycentric surface coordinate system with $r+s+t=1$ and let $Q$ denote the volumetric extension with $q=0$ at the top surface and $q=1$ at the bottom of the prism. Then a set $\mathbf{P}^{6}$ of degree one $C^{0}$-continuous shape functions can be constructed as presented in (16):

$$
\mathbf{P}^{6}(r, s, t, q)=[r(1-q), s(1-q), t(1-q), r q, s q, t q]
$$

This simple set of six linear shape functions controls the displacement of each prism vertex.

\section{$C^{1}$-Continuity at the Facial Surface}

In order to achieve $C^{1}$-continuity at the surface we first replace $P_{1}^{6} \ldots P_{3}^{6}$ by a set of nine functions derived from the well-known $\mathbf{N}^{9}$ introduced by $[4,10]$. Thus we arrive at a set of functions $\mathbf{P}^{12 \mathrm{a}}$ which allows to control the derivatives around the vertices on the facial surface. The resulting Hermite type barycentric polynomial functions for vertex $R$

$$
\begin{aligned}
& P_{1}^{12 a}=\left(r+r^{2} s+r^{2} t-r s^{2}-r t^{2}\right)(1-q) \\
& P_{2}^{12 a}=\left(r^{2} s+\frac{1}{2} r s t\right)(1-q) \\
& P_{3}^{12 a}=\left(r s^{2}+\frac{1}{2} r s t\right)(1-q)
\end{aligned}
$$

are depicted in figure 8 . The remaining functions $P_{4}^{12 a} \ldots P_{9}^{12 a}$ can be obtained by a cyclic permutation of $r, s$ and $t$.

The next step in accomplishing the $C^{1}$-continuity all over the facial surface is to control the derivatives at common vertices of adjacent triangles conformably. Therefore, we introduce the set of functions $\mathbf{P}^{12 \mathrm{~b}}$ which controls the derivatives at vertices with

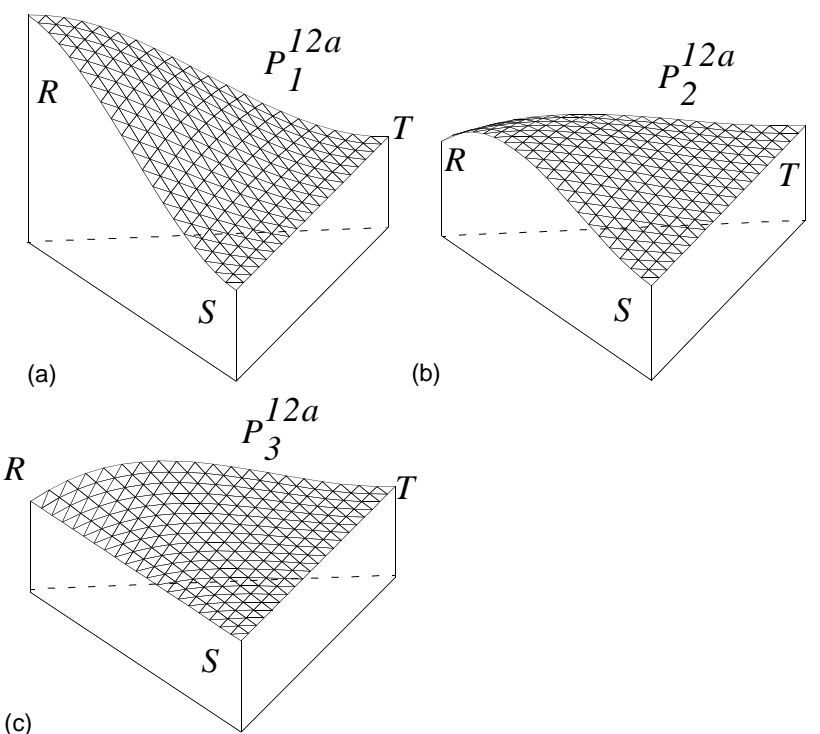

Figure 8: (a) Shape function controlling the displacement (b) Shape function controlling the derivative at $R$ in the direction of the surface triangle edge $t=0$

(c) Shape function controlling the derivative at $R$ in the direction of the surface triangle edge $s=0$

respect to the underlying global parametrization in $u$ and $v$ rather than in the direction of the triangle edges. The $\mathbf{P}^{12 b}$ follow from a transform of the $\mathbf{P}^{12 a}$

$$
\begin{aligned}
& P_{1}^{12 b}=P_{1}^{12 a} \\
& P_{2}^{12 b}=c_{3} P_{2}^{12 a}-c_{2} P_{3}^{12 a} \\
& P_{3}^{12 b}=-b_{3} P_{2}^{12 a}+b_{2} P_{3}^{12 a}
\end{aligned}
$$

with

$$
\frac{1}{2 \Delta}\left[\begin{array}{lll}
b_{1} & c_{1} & a_{1} \\
b_{2} & c_{2} & a_{2} \\
b_{3} & c_{3} & a_{3}
\end{array}\right]=\left[\begin{array}{lll}
u_{1} & u_{2} & u_{3} \\
v_{1} & v_{2} & v_{3} \\
1 & 1 & 1
\end{array}\right]^{-1}
$$

and

$$
2 \Delta=\left(-u_{1} v_{2}+u_{1} v_{3}+v_{1} u_{2}-v_{1} u_{3}-u_{2} v_{3}+u_{3} v_{2}\right)
$$

where $u_{i}$ and $v_{i}$ denote the coordinates of the vertices of the surface triangle. As an example, figure 9 shows the resulting $P_{2}^{12 b}$ interpolation function defined over a one-ring triangulation whose center vertex has valence six.

The contour line representation in figure $9 \mathrm{~b}$ illustrates that although providing $C^{1}$-continuity at vertices the $\mathbf{P}^{12 \mathrm{~b}}$ still suffer from discontinuities between adjacent elements. In finite element analysis, rational blend functions are used to tackle this problem (see e.g. [23]). The following rational $e$ functions allow us to control the cross-boundary derivatives independently of the displacements and derivatives at the vertices.

They are initially defined for the trivariate barycentric setting as follows:

$$
\begin{gathered}
e_{1}=\frac{r s^{2} t^{2}(1+r)}{(r+s)(r+t)}, \quad e_{2}=\frac{r^{2} s t^{2}(1+s)}{(s+t)(s+r)} \\
e_{3}=\frac{r^{2} s^{2} t(1+t)}{(t+r)(t+s)}
\end{gathered}
$$




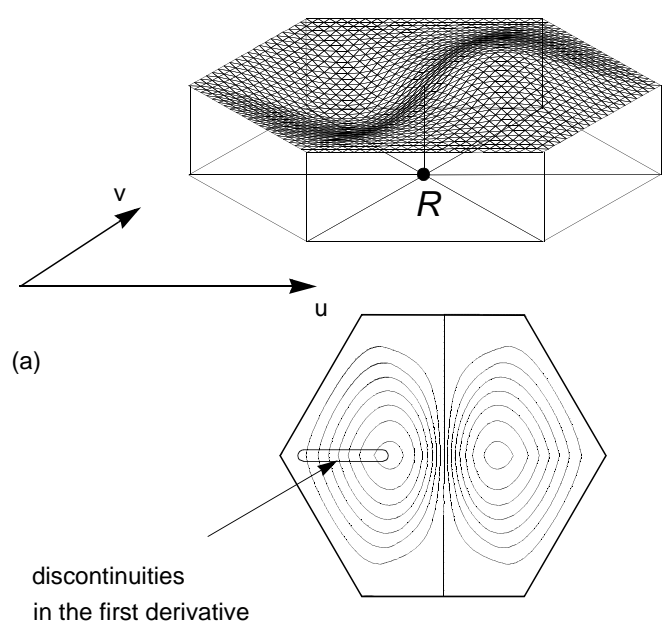

(b)

Figure 9: (a) One-ring of prism elements as represented by the $\mathbf{P}^{12 b}$ interpolation functions (b) Resulting contour lines

Figure 10 depicts a linear volumetric tensor product extension of $e^{1}$ by a multiplication with $(1-q)$. The resulting function controls the cross-boundary derivative at the edge $r=0$.

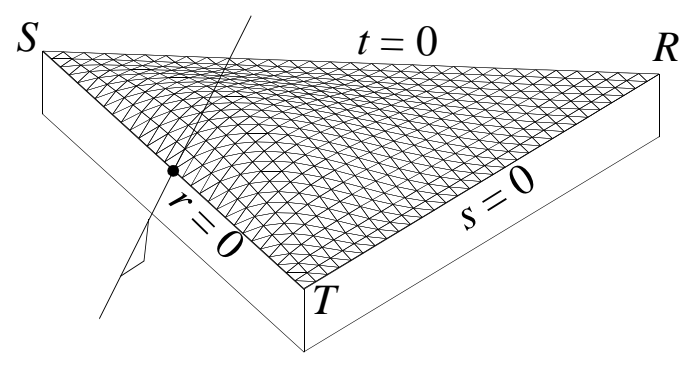

Figure 10: (a) Rational blend function used to control the cross-boundary derivative at edge $r=0$

A straightforward method to develop $C^{1}$-continuous surfaces is to integrate the required weights of the $e_{i}$ functions into the FEM problem as additional variables. Although being popular $[4,10]$, a major disadvantage of this approach is the increase of the overall degrees of freedom of the global system leading to higher computational costs. Further, various special cases have to be dealt with during the finite element assembly.

Therefore, we developed the following $\mathbf{N}^{9^{*}}$ interpolation functions for the prism surface according to [23]. Instead of introducing the weights of the $e$ functions as DOFs they are computed from the cross-boundary derivatives of the $\mathbf{N}^{9}$ functions at the vertex positions. We start with the normal derivative across each edge defined as

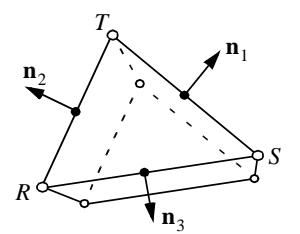

$$
\begin{aligned}
& \frac{\partial}{\partial \mathbf{n}_{1}}=\frac{l_{1}}{4 \Delta}\left[\frac{\partial}{\partial s}+\frac{\partial}{\partial t}-2 \frac{\partial}{\partial r}+\mu_{1}\left(\frac{\partial}{\partial t}-\frac{\partial}{\partial s}\right)\right] \\
& \frac{\partial}{\partial \mathbf{n}_{2}}=\frac{l_{2}}{4 \Delta}\left[\frac{\partial}{\partial t}+\frac{\partial}{\partial r}-2 \frac{\partial}{\partial s}+\mu_{2}\left(\frac{\partial}{\partial r}-\frac{\partial}{\partial t}\right)\right] \\
& \frac{\partial}{\partial \mathbf{n}_{3}}=\frac{l_{3}}{4 \Delta}\left[\frac{\partial}{\partial r}+\frac{\partial}{\partial s}-2 \frac{\partial}{\partial t}+\mu_{3}\left(\frac{\partial}{\partial s}-\frac{\partial}{\partial r}\right)\right]
\end{aligned}
$$

where the $l_{i}$ denote the lengths of the triangle edges, $\Delta$ the surface area and $\mu_{i}$ the geometric parameters

$$
\mu_{1}=\frac{l_{3}^{2}-l_{2}^{2}}{l_{1}^{2}} \quad \mu_{2}=\frac{l_{1}^{2}-l_{3}^{2}}{l_{2}^{2}} \quad \mu_{3}=\frac{l_{2}^{2}-l_{1}^{2}}{l_{3}^{2}} .
$$

As a next step we normalize the slopes of the $e_{i}$ to yield the $e_{i}^{\prime}$ prism functions with unit cross-boundary derivatives

$$
\begin{aligned}
\frac{\partial}{\partial \mathbf{n}_{1}} e_{1}(0,0.5,0.5) & =\frac{l_{1}}{8 \Delta} \\
\frac{\partial}{\partial \mathbf{n}_{2}} e_{2}(0.5,0,0.5) & =\frac{l_{2}}{8 \Delta}
\end{aligned} \quad \mathbf{e}^{\prime}(r, s, t, q)=\left[\begin{array}{c}
\frac{8 \Delta e_{1}}{l_{1}} \\
\frac{8 \Delta e_{2}}{l_{2}} \\
\frac{\partial}{\partial \mathbf{n}_{3}} e_{3}(0.5,0.5,0)=\frac{l_{3}}{8 \Delta}
\end{array} \quad[(1-q)\right.
$$

In order to obtain the $C^{1}$-continuous $\mathbf{P}^{12}$ interpolation functions we compute the average of the corresponding cross-boundary derivative $\frac{\partial}{\partial \mathbf{n}_{i}}$ at the endpoints of each edge $i$ of the surface triangle for the first nine components of $\mathbf{P}^{12 b}$ :

$$
\mathbf{Y}=\frac{1}{2}\left[\begin{array}{c}
\frac{\partial}{\partial \mathbf{n}_{1}} \mathbf{P}_{1 \ldots 9}^{12 b}(0,1,0,0)+\frac{\partial}{\partial \mathbf{n}_{1}} \mathbf{P}_{1 \ldots 9}^{12 b}(0,0,1,0) \\
\frac{\partial}{\partial \mathbf{n}_{2}} \mathbf{P}_{1 \ldots 9}^{12 b}(1,0,0,0)+\frac{\partial}{\partial \mathbf{n}_{2}} \mathbf{P}_{1 \ldots 9}^{12 b}(0,0,1,0) \\
\frac{\partial}{\partial \mathbf{n}_{3}} \mathbf{P}_{1 \ldots 9}^{12 b}(1,0,0,0)+\frac{\partial}{\partial \mathbf{n}_{3}} \mathbf{P}_{1 \ldots 9}^{12 b}(0,1,0,0)
\end{array}\right] .
$$

After computing the cross-boundary derivative at the edge midpoints of the first nine components of $\mathbf{P}^{12 b}$

$$
\mathbf{Z}=\left[\begin{array}{l}
\frac{\partial}{\partial \mathbf{n}_{1}} \mathbf{P}_{1 \ldots 9}^{12 b}(0,0.5,0.5,0) \\
\frac{\partial}{\partial \mathbf{n}_{2}} \mathbf{P}_{1 \ldots 9}^{12 b}(0.5,0,0.5,0) \\
\frac{\partial}{\partial \mathbf{n}_{3}} \mathbf{P}_{1 \ldots 9}^{12 b}(0.5,0.5,0,0)
\end{array}\right]
$$

we are now able to define a vector of prism shaped interpolation functions featuring global $C^{1}$-continuity at the top surface

$$
\mathbf{P}^{12}=\mathbf{P}^{12 b}+e^{\prime}(\mathbf{Y}-\mathbf{Z} \mid \mathbf{0}) .
$$

\section{Element Node Vector}

In contrast to the interpolation of the vector valued displacement with the $\mathbf{P}^{12}$ functions the $\mathbf{P}^{6}$ linear functions are used to interpolate the scalar variable representing the pressure in the mixed formulation.

The upper definitions yield the following node or weight vector for an element $m$

$$
[\hat{\mathbf{U}} \mid \hat{\mathbf{P}}]^{(m)}=\left[\hat{\mathbf{U}}_{x}^{(m)}, \hat{\mathbf{U}}_{y}^{(m)}, \hat{\mathbf{U}}_{z}^{(m)}, \hat{\mathbf{P}}^{(m)}\right]
$$


with the weight subvectors $\hat{\mathbf{U}}_{x}^{(m)}, \hat{\mathbf{U}}_{y}^{(m)}$ and $\hat{\mathbf{U}}_{z}^{(m)}$ for the interpolation of displacements in the corresponding coordinate directions and the weights $\hat{\mathbf{P}}^{(m)}$ for the interpolation of pressure:

$$
\begin{aligned}
\hat{\mathbf{U}}_{x}^{(m)} & =\left[x_{1} \frac{\partial U_{x_{1}}}{\partial u} \frac{\partial U_{x_{1}}}{\partial v} x_{2} \frac{\partial U_{x_{2}}}{\partial u} \frac{\partial U_{x_{2}}}{\partial v} x_{3} \frac{\partial U_{x_{3}}}{\partial u} \frac{\partial U_{x_{3}}}{\partial v} x_{4} x_{5} x_{6}\right] \\
\hat{\mathbf{U}}_{y}^{(m)} & =\left[y_{1} \frac{\partial U_{y_{1}}}{\partial u} \frac{\partial U_{y_{1}}}{\partial v} y_{2} \frac{\partial U_{y_{2}}}{\partial u} \frac{\partial U_{y_{2}}}{\partial v} y_{3} \frac{\partial U_{y_{3}}}{\partial u} \frac{\partial U_{y_{3}}}{\partial v} y_{4} y_{5} y_{6}\right] . \\
\hat{\mathbf{U}}_{z}^{(m)} & =\left[z_{1} \frac{\partial U_{z_{1}}}{\partial u} \frac{\partial U_{z_{1}}}{\partial v} z_{2} \frac{\partial U_{z_{2}}}{\partial u} \frac{\partial U_{z_{2}}}{\partial v} z_{3} \frac{\partial U_{z_{3}}}{\partial u} \frac{\partial U_{z_{3}}}{\partial v} z_{4} z_{5} z_{6}\right] \\
\hat{\mathbf{P}}^{(m)} & =\left[p_{1} p_{2} p_{3} p_{4} p_{5} p_{6}\right]
\end{aligned}
$$

\section{Initial surface fairing}

As we are computing a smooth displacement field to the original configuration we require a smooth presurgical facial shape. Therefore, we have to find estimates for an initial node vector used to define the pre-surgical smooth shape.

In the proposed setting, it would be straightforward to use the finite element approach to compute a $C^{1}$-surface with minimal energy with respect to a surface energy measure $[4,10]$. This, however, would require an additional FEM solving step in advance. In order to reduce the computational costs we approximate the nodal weights corresponding to the derivatives of the initial surface by means of finite differences. Alternatively, subdivision schemes such as [13] and discrete fairing methods could be used to find estimates.

\subsection{Matrix Formulation}

Using the sets of shape functions $\mathbf{P}^{12}$ and $\mathbf{P}^{6}$ together with the corresponding weight vectors (29) yields

$$
\begin{aligned}
& \mathbf{U}^{(m)}(u, v, q)=\left[\begin{array}{c}
U_{x}^{(m)}(u, v, q) \\
U_{y}^{(m)}(u, v, q) \\
U_{z}^{(m)}(u, v, q)
\end{array}\right]= \\
& {\left[\begin{array}{c|c|c}
\mathbf{P}^{12(m)} & 0 & 0 \\
0 & \mathbf{P}^{12(m)} & 0 \\
0 & 0 & \mathbf{P}^{12(m)}
\end{array}\right]\left[\begin{array}{l}
\hat{\mathbf{U}}_{x}^{(m)} \\
\hat{\mathbf{U}}_{y}^{(m)} \\
\hat{\mathbf{U}}_{z}^{(m)}
\end{array}\right]=\mathbf{H}^{(m)} \hat{\mathbf{U}}^{(m)}}
\end{aligned}
$$

for the interpolation of displacements and

$$
p^{(m)}(u, v, q)=\mathbf{P}^{6} \hat{\mathbf{P}}^{(m)}=\mathbf{H}_{p} \hat{\mathbf{P}}^{(m)}
$$

for the interpolation of pressure within an element $m$. Note that in contrast to the $\mathbf{P}^{12}$ the linear shape functions $\mathbf{P}^{6}$ are independent of the element geometry and therefore can be written as $\mathbf{H}_{p}$ instead of $\mathbf{H}_{p}^{(m)}$.

By introducing an operator matrix of the first order derivatives used in the definition of the strain vector the deviatoric strain (10) can be formulated as

$$
\boldsymbol{\varepsilon}^{\prime}=\frac{1}{3}\left[\begin{array}{ccccc}
2 & -1 & -1 & \\
-1 & 2 & -1 & 0 \\
-1 & -1 & 2 & & \\
& & 3 & \\
& & & 3 & 3
\end{array}\right]\left[\begin{array}{llll}
\frac{\partial}{\partial X} & & \frac{\partial}{\partial Y} & \frac{\partial}{\partial Z} \\
\frac{\partial}{\partial Y} & \frac{\partial}{\partial X} & \frac{\partial}{\partial Z} \\
& \frac{\partial}{\partial Z} & \frac{\partial}{\partial Y} \frac{\partial}{\partial X}
\end{array}\right]^{T} \mathbf{H}^{(m)} \hat{\mathbf{U}}^{(m)}=\mathbf{B}_{D}^{(m)} \hat{\mathbf{U}}^{(m)}
$$

and for the volumetric strain (11) we find analogously

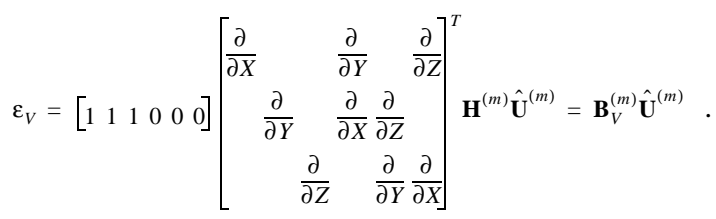

Using the Galerkin projections (31), (32) and (33) to express (14) and (15) yields a matrix formulation of the problem. Integrating over each element using Gaussian quadrature and summing up the contribution of each element into one global system of linear equations yields

$$
\mathbf{K U}=\mathbf{0}
$$

where $\mathbf{K}$ denotes the global stiffness matrix and $\mathbf{U}$ represents the global weight vector we are solving for. Invoking the prescribed displacement boundary conditions yields a non-trivial solution of (34). Either a conjugate gradient approach or a direct solver for the case of total or near incompressibility is used to solve (34). An elaboration of the structure and derivation of (34) is given in [1].

\section{RESULTS AND VALIDATION}

\section{Error Evaluation and Visualization}

In order to validate the prototype and to obtain both a quantitative and a visual impression of the quality of simulation, we quantitatively compare the post-surgical facial surface with the surface resulting from simulation. Therefore, we first register both geometries as described in section Section 2.1 and then approximate the error at each surface coordinate by projecting the radial distance between the surfaces onto a local normal given by the average of both surface normals at the intersection points. The error visualization as depicted in figure 11a is obtained by pseudo-coloring these distance values at the facial areas affected by surgery. Non-matchable regions like hair, eyebrows and eyes are set to zero.

(a)

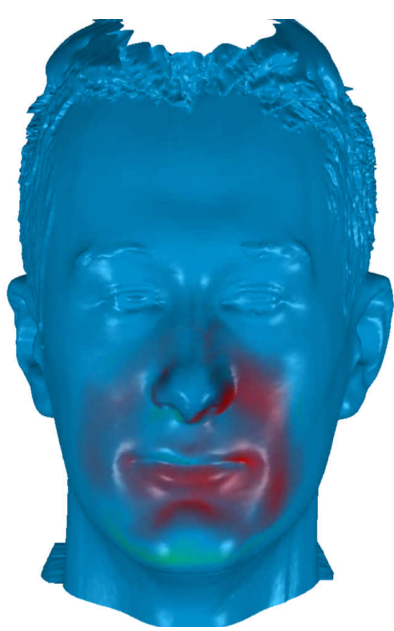

Š $-3 \mathrm{~mm}$

$0 \mathrm{~mm}$

Figure 11: Visualization of the error distribution in terms of the radial distance between simulated and real post-surgical surface (a) and the color map used for pseudo-coloring (b)

The pseudo-coloring is performed by means of the color map in figure $11 \mathrm{~b}$. Positive values indicate the predicted surface lying in front of the post-surgical face whereas red colors illustrate the contrary. Errors at the side of the nose common to all cases must be attributed to the radial measuring set-up which tends to overemphasize errors at places where the surface happens to be of nearly the same orientation as the radial direction of measure- 
ment. Further, errors at the cheekbones are due to swelling. The quantitative simulation error given as a volumetric difference in figures 12 and 13 is computed by integrating the absolute values of the distances over the surface of interest.

\section{Sources of Error}

According to clinicians, the prediction performance of our system is excellent. However, before presenting individual cases some general sources of errors deserve discussion. Detectable minor differences between prediction and real result may be contributed to the following factors:

- The exact correspondence of the three-dimensional metric displacements such as depicted in figure 4 with the actual surgery is of paramount importance for the quality of simulation.

- Slight deviations may result from the state of swelling which can take up to one year. Further, differences in facial muscular activity between pre- and post-operative picture and laser range scan are unavoidable.

- The matching of the post-surgical laser range scan and the result of the simulation needed for the quantitative evaluation is prone to errors as appropriate reference points can only be found in unaltered regions of the face. In addition, head alignment and motion artifacts can distort the quality of the laser range scans.

- Overemphasis of certain facial structures in the resulting renderings of the simulation is due to differences in the lighting conditions of simulation and post-surgical photographs.

\section{Individual Patients}

The results of the simulated correction of the short face syndrome of our example patient are depicted in figure 12. He suffers from a retrodisplacement of the maxilla and mandible (upper and lower jaw) in combination with a deep bite because of a predominantly horizontal growth pattern of the bases of the jaws in relation to the skull base.

The correspondence of simulation and real surgery is exceptional. The profile lines are given in figure $12 \mathrm{~d}$, where blue, green and red represent pre-surgical, simulated and post-surgical situation respectively. Minor deviations can only be observed in the region of the mouth. In addition, the frontal error visualization reveals artifacts due to swelling.

In the second case (figure 13) a retromaxillism with dished-in midface had to be cured. This malformation is caused by an insufficient anterior (sagittal) growth of the maxilla in combination with a slightly overprojected mandible. As a consequence, upper lip, nose and especially paranasal tissues are not sufficiently advanced. Further, the chin is far to prominent.

Again, there is little deviation between simulation and real outcome. The main error at the lower chin is caused by unaligned scanning positions.

\section{CONCLUSIONS AND FUTURE WORK}

We have presented a framework for facial surgery simulation which combines the quality of a $C^{1}$-continuous facial surface with the accuracy of volumetric finite element simulation. Further, we have given a proof of concept by comparing simulation and real surgery. Both the error analysis and the clinician's evaluation of the results demonstrate the superiority of our vision for surgical planning over the conventional methods.

In spite of the performance of the framework future work will comprise the following aspects: the restriction to $C^{0}$-continuity in the interior might have effects on the volumetric behavior of the tissue. An extension of the system to $C^{1}$-continuous tetrahedral elements giving additional topological freedom is currently being investigated. Furthermore, in order to comply with results of biomechanical studies, we plan to include non-linear elasticity into the model. Since, however, the additional computational costs are enormous the resulting benefits have to be evaluated thoroughly. Finally, adaptive multigrid methods in combination with hierarchical bases could substantially speed-up the simulation.

\section{ACKNOWLEDGEMENT}

This work was partially supported by the Swiss National Science Foundation (SNSF) under grant no. 2153-049247.96/1.

We thank our example patient Dario Dobranic for his patience and willingness to participate in the study.

\section{REFERENCES}

[1] K.-J. Bathe. Finite element procedures in engineering analysis. Prentice Hall, Englewood Cliffs, 1982.

[2] M. Bro-Nielsen and S. Cotin. "Real-time volumetric deformable models for surgery simulation using finite elements and condensation." In Proc. EUROGRAPHICS'96, 1996.

[3] A. E. Carlotti, P. H. Aschaffenburg, and S. A. Schendel. "Facial changes associated with surgical advancement of the lip and maxilla." Journal of Oral and Maxillofacial Surgery, 44:593, 1986.

[4] G. Celniker and D. Gossard. "Deformable curve and surface finite elements for free-form shape design.” In T. W. Sederberg, editor, Computer Graphics (SIGGRAPH '91 Proceedings), volume 25, pages 257-266, July 1991.

[5] X. Q. Deng. A Finite Element Analysis of Surgery of the Human Facial Tissues. Ph.D. thesis, Columbia University, 1988.

[6] L. G. Farkas. Anthropometry of the Head and Face. Raven Press, 2nd edition, 1994

[7] B. Fromm and M. Lundberg. "The soft-tissue facial profile before and after surgical correction of mandibular protrusion." Acta Odontologica Scandinavia, 28:157, 1970.

[8] Y. C. Fung. Biomechanics: mechanical properties of living tissues. Springer, 2nd edition, 1993.

[9] E. Keeve, S. Girod, P. Pfeifle, and B. Girod. "Anatomy-based facial tissue modeling using the finite element method." In Proc of IEEE Visualization'96, Nov. 1996.

[10] R. M. Koch, M. H. Gross, F. R. Carls, D. F. von Büren, G. Fankhauser, and Y. I. H. Parish. "Simulating facial surgery using finite element models." In SIGGRAPH 96 Conference Proceedings, Annual Conference Series, pages 421-428. ACM SIGGRAPH, Addison Wesley, Aug. 1996. held in New Orleans, Louisiana, 4-9 August 1996.

[11] W. Larrabee. "A finite element model of skin deformation. I biomechanics of skin and soft tissue: A review." In Laryngoscope, pages 399-405. 1986.

[12] Y. Lee, D. Terzopoulos, and K. Waters. "Realistic face model ing for animation." In R. Cook, editor, SIGGRAPH 95 Conference Proceedings, Annual Conference Series, pages 55-62. ACM SIGGRAPH, Addison Wesley, Aug. 1995. held in Los Angeles, California, 06-11 August 1995.

[13] C. Loop. "Smooth spline surfaces over irregular meshes." In A. Glassner, editor, Proceedings of SIGGRAPH '94 (Orlando, Florida, July 24-29, 1994), Computer Graphics Proceedings, Annual Conference Series, pages 303-310. ACM SIGGRAPH, ACM Press, July 1994. ISBN 0-89791-667-0.

[14] W. E. Lorensen and H. E. Cline. "Marching cubes: A high resolution 3D surface construction algorithm.” In M. C. Stone, editor, Computer Graphics (SIGGRAPH '87 Proceedings), volume 21, pages 163-169, July 1987.

[15] F. Parke and K. Waters. Computer Facial Animation. A K Peters, 1993. ISBN 1-56881-014-8.

[16] F. I. Parke. "Parameterized models for facial animation." IEEE Computer Graphics and Applications, 2:61-68, Nov. 1982.

[17] S. Pieper. CAPS: Computer-Aided Plastic Surgery. Ph.D. thesis, Massachusetts Institute of Technology, 1991 
[18] W. H. Press, S. A. Teukolsky, W. T. Vetterling, and B. P. Flannery. Numerical Recipes in C: The Art of Scientific Computing (2nd ed.). Cambridge University Press, Cambridge, 1992. ISBN $0-521-43108-5$

[19] S.H.M. Roth: Bernstein-Bézier Representations for Facial Surgery Simulation. PhD thesis No. 14531, Department of Computer Science, ETH Zürich, 2002.

[20] W. J. Schroeder, J. A. Zarge, and W. E. Lorensen. "Decimation of triangle meshes." In Proceedings of SIGGRAPH '92, pages 65-70. ACM SIGGRAPH, 1992.
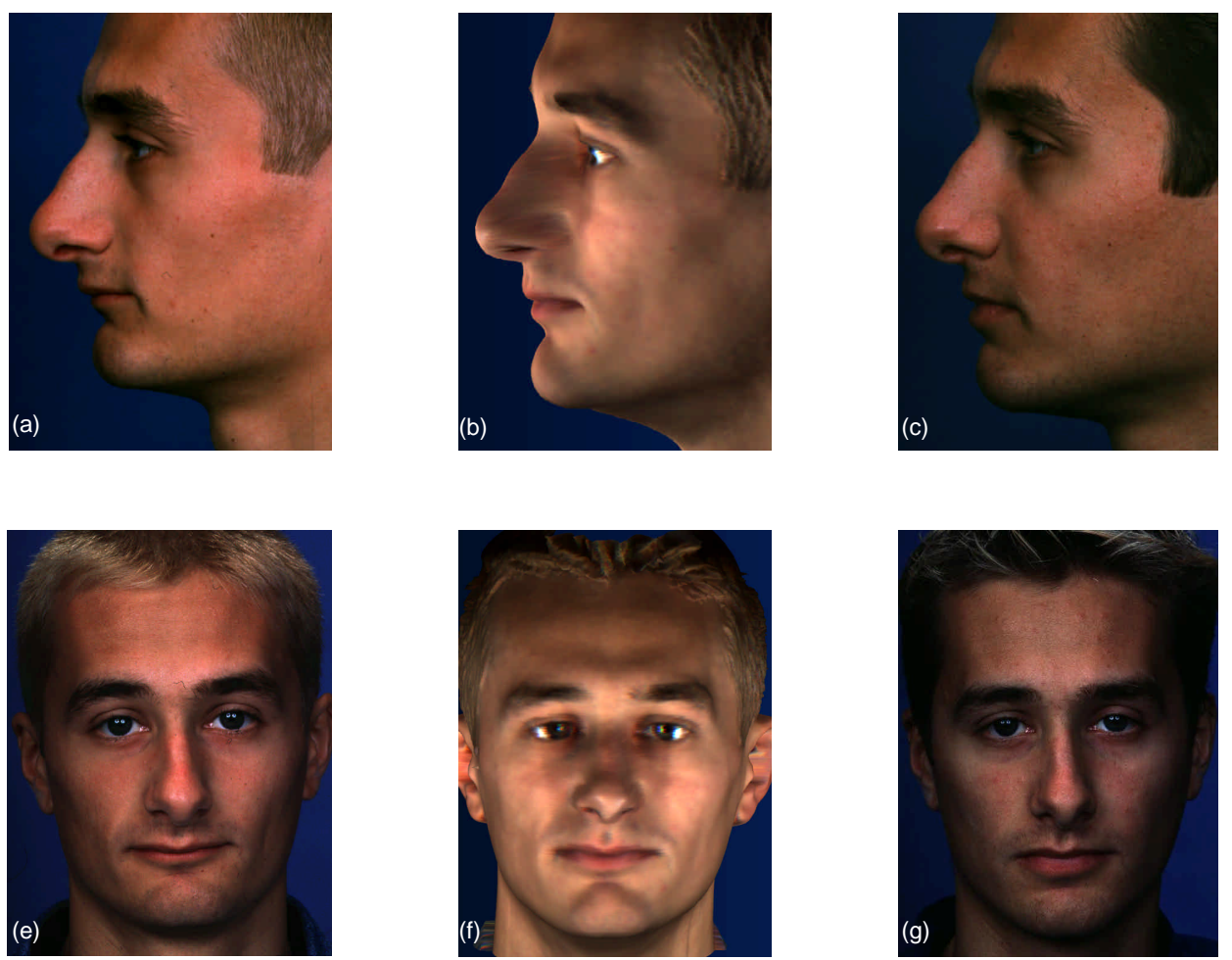

Figure 12: Profiles: (a) pre-surgical, (b) predicted, (c) post-surgical, (d) profile lines

Portraits: (e) pre-surgical, (f) predicted, (g) post-surgical, (h) error visualization
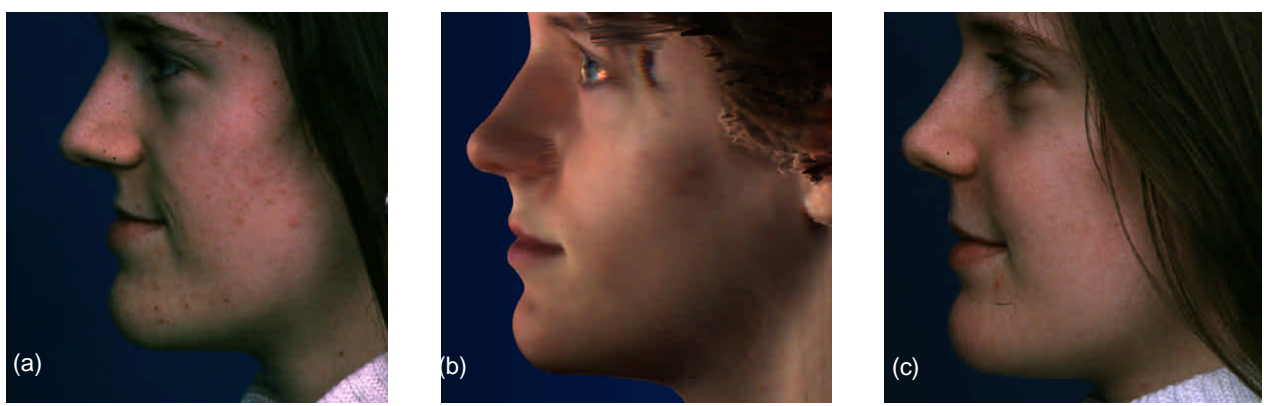

(d)

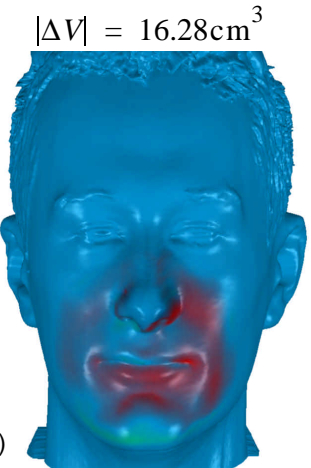

(h)
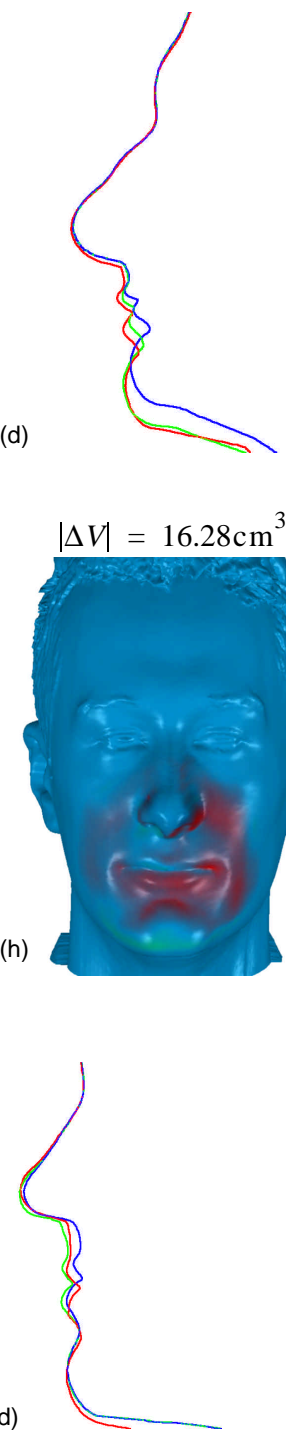

$|\Delta V|=13.12 \mathrm{~cm}^{3}$
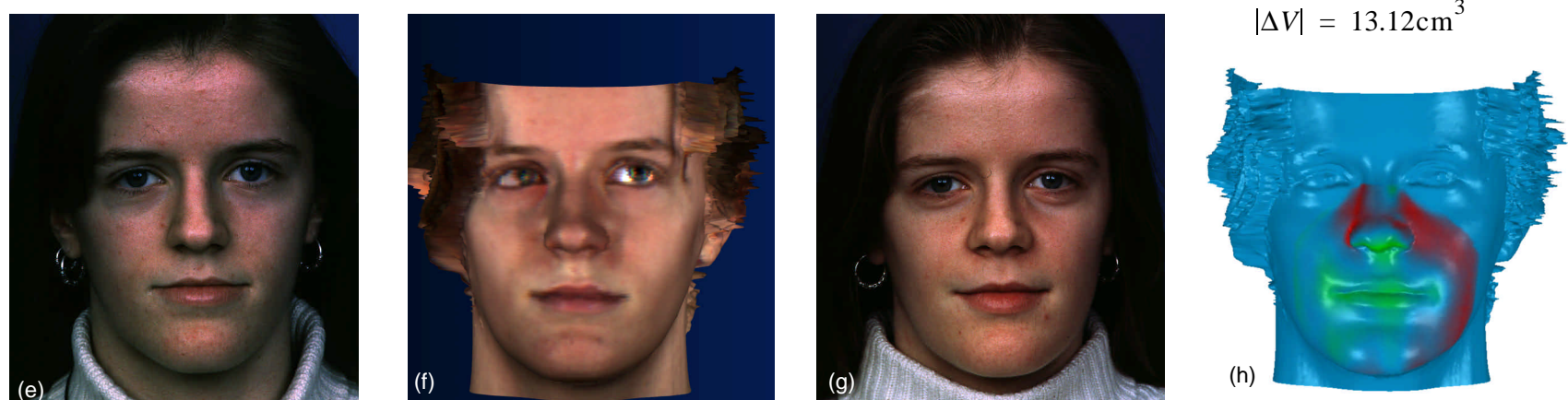

Figure 13: Profiles: (a) pre-surgical, (b) predicted, (c) post-surgical, (d) profile lines

Portraits: (e) pre-surgical, (f) predicted, (g) post-surgical, (h) error visualization 